Brit. Heart F., 1969, 31, 112.

\title{
Clinical and Pharmacological Factors Associated with Pacemaker Latency and Incomplete Pacemaker Capture
}

\author{
ARTHUR J. MOSS AND SIDNEY GOLDSTEIN
}

From the Department of Medicine, University of Rochester School of Medicine and Dentistry, Strong Memorial Hospital, and the Rochester General Hospital, Rochester, New York, U.S.A.

The myocardial response to pacemaker stimuli is usually all or none once the myocardial threshold is exceeded. With fixed rate pacemakers, which function as artificial parasystolic foci (Núñez-Dey, Zalter, and Eisenberg, 1962), the heart is captured by the regularly generated stimuli so long as the pacemaker is functional, properly positioned, and delivers its impulse outside the refractory period of the intrinsic electrical activity of the heart (Linenthal and Zoll, 1962). In addition, the time interval from the pacemaker impulse to initiation of the ventricular response is generally short, and the stimulus artefact is usually fused with the resultant QRS complex. If the pacemaker stimulus is not coincident with the pacemaker-induced depolarization, the phenomenon may be described as pacemaker latency.

During the management of 129 symptomatic heart block patients with either temporary transvenous or implanted transvenous and transthoracic pacemakers, four'varieties of incomplete pacemaker capture were observed in five patients. In one of these patients, a significant latency in activation was noted. These observations, which form the substance of this report, bear on certain theories relating to the irregular expression of ventricular parasystolic foci.

\section{SUBJECTS AND MethodS}

Permanent transthoracic pacemakers were implanted in 28 patients using the technique of Chardack, Gage,

Received April 8, 1968.

* Supported in part by grants-in-aid from the U.S. Public Health Service, the Genesee Valley Heart Association, the Hochstetter Fund, and the Commonwealth Fund. and Greatbatch (1960). Permanent transvenous pacemakers were implanted in 41 patients, and the electrode tip was positioned in the right ventricular apex as described by the Medtronic manufacturer (1965). Temporary transvenous pacemakers were inserted into 60 patients with the electrode tip positioned in the right ventricular apex. Bipolar pacemaker catheters and external Medtronic generators * were used for temporary pacing. Implantation of all transvenous catheter systems was done under image intensification fluoroscopy, and proper catheter tip position was verified by consistent pacing below 2.0 milliamps. Final generator settings were above twice threshold.

\section{RESULTS}

Group A: Pacemaker Latency. One patient was noted to have prolonged intervals between the pacemaker artefact and the QRS response. This latency was variable and was associated with an incomplete capture of the ventricle, including a Wenckebach-type ventricular response.

Case 1. A 66-year-old man with known coronary heart disease required temporary transvenous pacemaker therapy for symptomatic complete heart block. While being paced at a rate of $75 / \mathrm{min}$., runs of ventricular tachycardia developed and the pacemaker rate was increased to 100 beats/min. Episodic ventricular tachycardia continued, and intravenous lignocaine was administered in doses of $1-3 \mathrm{mg} . / \mathrm{min}$., and in supplemental boli of $25-50 \mathrm{mg}$. Various types of pacemaker latency and incomplete ventricular capture developed during this unstable arrhythmic period (Fig. 1 and 2). Ventricular tachycardia and fibrillation recurred, necessitating direct current countershock. Terminally, asystole

* Medtronic External Pulse Generator, Model 5800; Catheter Electrode, Model 5821; Mfg. Medtronic, Inc., Minneapolis, Minn., U.S.A. 


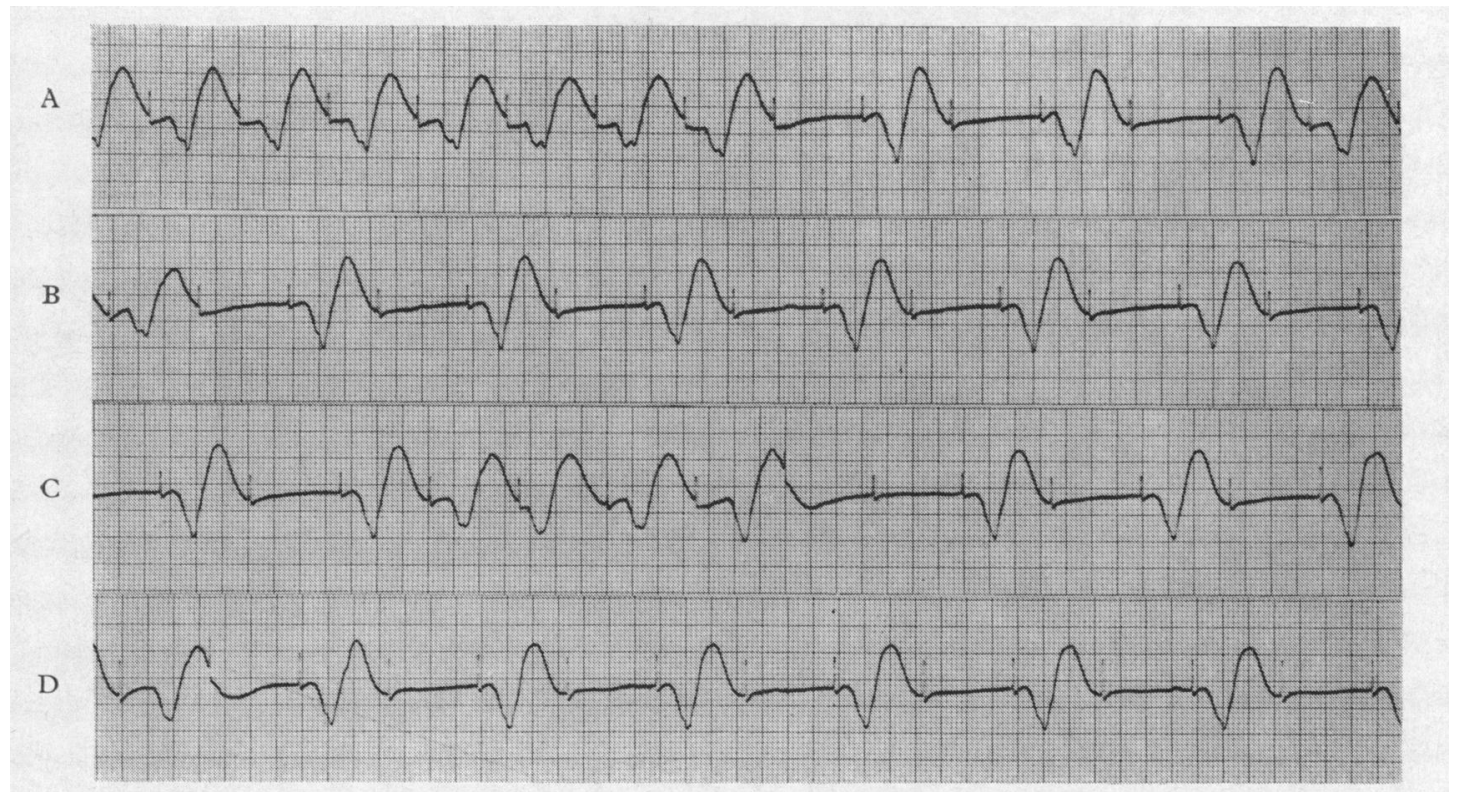

FIG. 1.-Pacemaker latency and incomplete pacemaker capture during lignocaine administration. Fixed transvenous pacemaker rate at 85 beats per minute. The Q-T interval is prolonged to $560 \mathrm{msec}$. or greater. (Case 1, see text for details.) Paper speed of this and all subsequent tracings is $25 \mathrm{~mm}$. per second. (A) In the initial portion of the tracing, the pacemaker latency is $160 \mathrm{msec}$. and the pacemaker conducts $1: 1$. In the latter portion, 2:1 capture is present and the latency of the pacemaker conducted beats has been reduced to 120 msec. (B) Fixed 2:1 pacemaker capture with a 120 msec. latency in the conducted beats. (C) A variable type of pacemaker latency and capture. Beginning with the fifth pacemaker stimulus (stimulusresponse interval $60 \mathrm{msec}$.), progressively longer stimulus-response intervals ensue (160 and $260 \mathrm{msec}$.) and the eighth and ninth pacemaker stimuli do not capture the heart. Thereafter, $2: 1$ capture is present. (D) Though the pacemaker stimulus has been increased in intensity, the first pacemaker stimulus is still followed by a prolonged interval $(260 \mathrm{msec}$.) before the onset of the QRS depolarization. The remaining portion of this strip reveals $2: 1$ capture with a $100 \mathrm{msec}$. latency in the conducted beats.

developed and the myocardium did not respond to pacemaker stimulation. Necropsy revealed that the catheter was well positioned in the right ventricular apex with the tip against the endocardium.

Group B: Isolated Dropped Pacemaker Beats. In 1928 Mobitz described a type of atrioventricular heart block characterized by an occasional dropped beat, but without $P-R$ interval prolongation in the remaining, normally conducted beats. An analogous type of incomplete capture was observed in one patient with a pacemaker rhythm; isolated dropped pacemaker beats occurred in the absence of significant pacemaker latency.

Case 2. A 73-year-old woman with an acute diaphragmatic myocardial infarction developed complete heart block and required implantation of a temporary transvenous pacemaker. Threshold for stimulation was 1.5 milliamps, and pacemaker stimulation was set at 6.0 milliamps. Her hospital course was complicated by recurrent episodes of ventricular tachycardia, requiring intravenous lignocaine drip, $1 \mathrm{mg}$./min. During lignocaine administration the threshold for stimulation pro- gressively increased to 6.0 milliamps and the pacemaker was set at 9.0 milliamps. All pacemaker stimuli did not capture the ventricle and an occasional dropped beat was recorded; with additional lignocaine, $2: 1$ pacemaker capture developed (Fig. 3). Repeated episodes of ventricular fibrillation necessitated direct current countershock, and terminally the myocardium became unresponsive to pacemaker stimulation. Necropsy revealed that the catheter was in good position, with the tip against the endocardium and wedged in the right ventricular apex under the trabeculae.

Group C: Two-to-one Pacemaker Capture. Twoto-one exit capture was recorded in 3 patients. In 2 it was observed during lignocaine treatment for ventricular irritability (Cases 1 and 2, Fig. 1-3); it was also seen in a third patient 24 hours after the implantation of a Medtronic transthoracic pacemaker. In this latter patient, a fixed rate pacemaker was initially set at twice threshold ( $2 \cdot 8$ milliamps) at a rate of $72 / \mathrm{min}$. Multifocal ventricular ectopic beats appeared during the immediate post-operative period, and control was achieved by increasing the rate of the pacemaker to $88 / \mathrm{min}$. Treatment with 


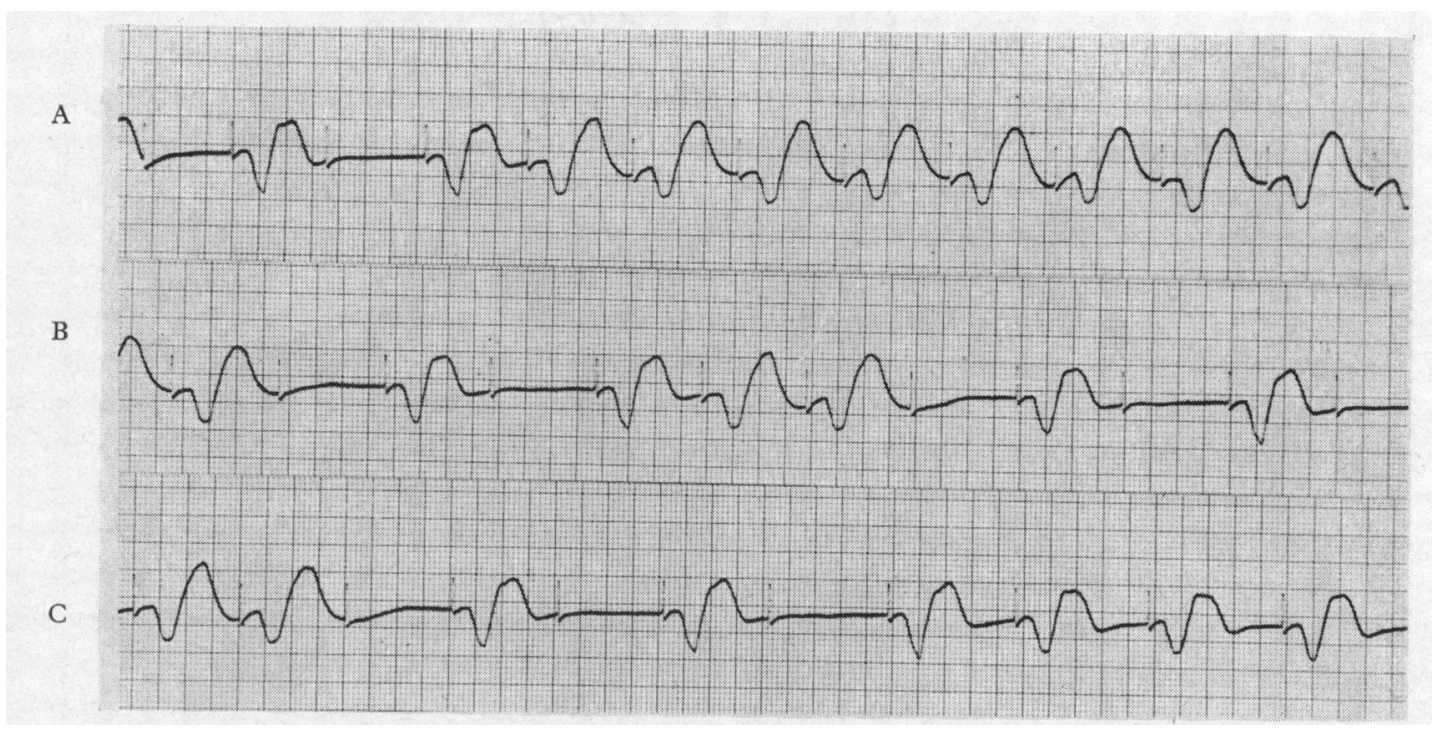

FIG. 2.-Various types of pacemaker latency and incomplete pacemaker capture during lignocaine administration and progressive slowing of the pacemaker rate from 91 to 60 beats per minute. The intensity of the pacemaker stimulus was maintained constant. Q-T interval is prolonged to $560 \mathrm{msec}$. or greater. (Case 1 , see text for details.) (A) As the pacemaker rate is slowed from 91 to 75 beats a minute, a $2: 1$ conduction pattern with stimulus-response intervals of $120 \mathrm{msec}$. changes to $1: 1$ conduction with increase in the stimulusresponse intervals to $140-150 \mathrm{msec}$. (B) Fixed pacemaker rate at 75 beats a minute. After an initial $2: 1$ capture of the ventricle, pacemaker beats 5-7 reveal progressively longer stimulus-response intervals from 120 to $150 \mathrm{msec}$; the 8 th pacemaker beat is blocked and 2:1 conduction ensues. Pacemaker beats 5-8 reveal a Wenckebach type of conduction latency, with the maximal increment of conduction delay between the first and second (pacemaker artefacts 5 and 6) conducted beats. (C) As the pacemaker rate is slowed from 75 to 60 beats a minute, a $2: 1$ conduction with stimulus-response intervals of $120-140 \mathrm{msec}$. changes to $1: 1$ conduction with decrease in stimulus-response intervals to about $100 \mathrm{msec}$.

procainamide, $500 \mathrm{mg}$. intramuscularly every 6 hours, was started. In this setting, a transient run of 2:1 pacemaker capture preceded the development of complete failure of the pacemaker to capture the ventricle. The pacemaker amplitude was increased to maximum ( 5 milliamps) and pacemaker domination was re-established.

Group D: High-grade Incomplete Pacemaker Capture. In 2 patients with implanted transvenous pacemakers, high-grade incomplete pacemaker capture developed 10-14 months after implantation. In both patients the pacemaker captured the ventricle only when the stimulus artefact fell in the terminal portion of the $T$ wave, i.e. during the socalled supernormal phase of excitability of a preceding depolarization (Fig. 4). In each case, the generator was tested, and battery deterioration had not taken place. However, the excitation threshold, which was originally less than $2 \cdot 0$ milliamps at the time of implantation, had increased above 5.0 milliamps. The catheter tips were in good position, and wire discontinuity was excluded by testing each electrode as a unipolar system. In both cases, com- plete capture of the ventricle was re-established by implanting a more powerful battery unit.

\section{Discussion}

All 5 patients with abnormalities in pacemaker stimulation had significant intrinsic myocardial disease. Three patients were receiving antiarrhythmic agents, and two had a documented rise in the threshold for stimulation at the time of the conduction disturbance. In 4 of the 5 patients the pacemaker stimuli were delivered to the endocardium by a transvenous system.

Multiple mechanisms may be responsible for the observed incomplete pacemaker capture. If the pacemaker stimulus delivered to the myocardium is reduced to or below excitation threshold, due either to malposition of the pacing electrode or to a decrease in the generator output, irregular or erratic pacing may ensue. In the 4 patients with transvenous units, the catheters appeared to be well positioned as judged by fluoroscopy, by an initial low threshold for stimulation, and by subsequent necropsies in 2 patients. Nevertheless, absolute constancy of electrode contact with the endocardium 
FIG. 3.-Transvenous pacemaker with isolated dropped pacemaker beats and 2:1 capture following lignocaine administration. (Case 2, see text for details.) (A) Oneto-one pacemaker activation of the ventricle before lignocaine administration. After each pacemaker stimulus, the QRS depolarization appears to start promptly with a slow, small deflection suggesting an initial intraventricular conduction disturbance. (B) An isolated dropped pacemaker beat during lignocaine administration. In the conducted pacemaker beats which follow each stimulus, the initial intraventricular conduction disturbance is more marked than before lignocaine administration (A). The time interval between the pacemaker stimulus and the onset of rapid depolarization is about $80 \mathrm{msec}$. The nonconducted fourth pacemaker stimulus is followed by a small deflection which may represent either a stimulus artefact or a localized myocardial depolarization process which does not activate the entire ventricle. (C) A run of 2:1 pacemaker capture during further lignocaine administration.

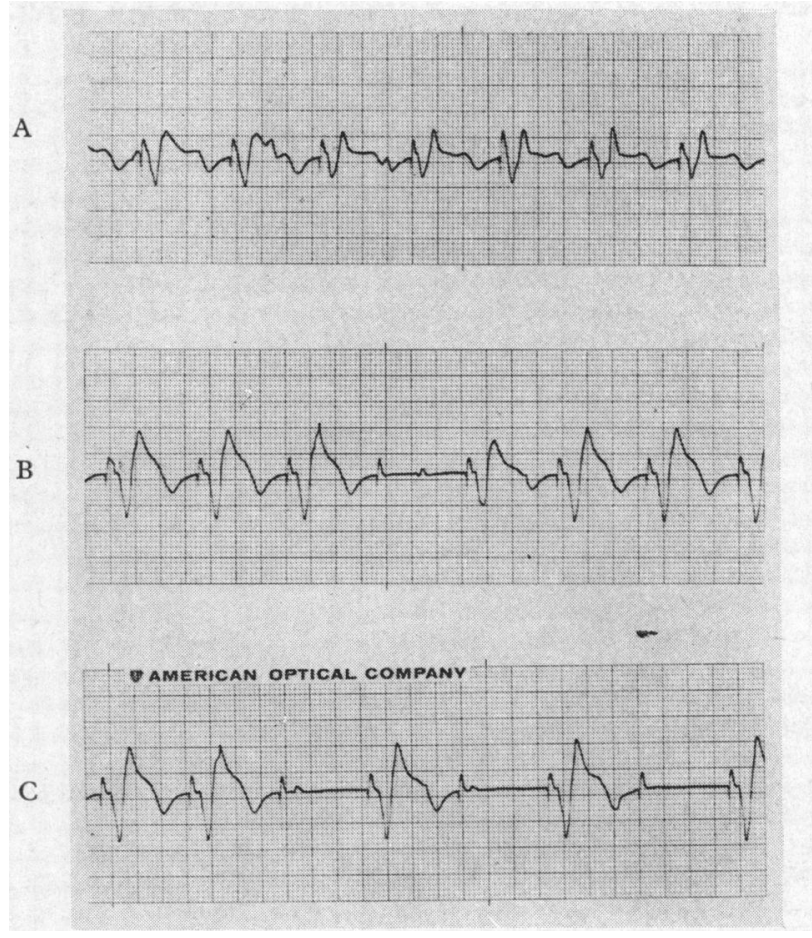

cannot be claimed. Thus, the occasional dropped beat in Case 2 may be due to inconstant contact of the electrode tip with the endocardium when the stimulus is just barely suprathreshold. However, transient 2:1 pacemaker capture was observed in one patient with electrodes implanted directly into the myocardium by the transthoracic approach, and inconstant electrode contact with the ventricle seems excluded in this patient. Generator output was checked in 2 of the 3 patients with implanted battery units, and the batteries were found to be normal at the time of the failure to pace. The external generator source in the remaining 2 patients with temporary transvenous units was not specifically checked for possible battery malfunction.

Pathological and pharmacological alterations in the electrophysiological properties of the myocardium seem to be the most likely explanation for latency and incomplete pacemaker capture in the first three cases (Groups A, B, C). These conduction disturbances were observed during the vigorous administration of antiarrhythmic agents in an attempt to control ventricular irritability. Two of the three patients were preterminal in a low output state, and incomplete pacemaker capture developed in the third patient during the immediate postoperative period after transthoracic implantation of epicardial electrodes. Lignocaine and procainamide administration as well as myocardial hypoxia prolong the refractory period, slow impulse conduction, and increase the excitation threshold of myocardial tissue (Hoffman and Cranefield, 1964). In Case 1 , the Q-T interval was very prolonged (560 msec.). As successive pacemaker stimuli fell within this abnormally increased Q-T interval (Fig. 1, C), an increasing stimulus-response latency became evident, due in part to a prolonged refractory period effect, and pacemaker stimuli failed to activate the ventricle. However, even during 2:1 conduction when the R-R intervals were long, latency in activation was still present. This latter finding indicates an abnormality in activation that is independent of rate.

The mechanism of the conduction disturbances in the aforementioned three patients, in whom antiarrhythmic agents were administered, appears to be quite different from the last two patients (Group D) in whom the problem was solved by using a more powerful generator. In this latter group, pacemaker capture of the ventricle occurred only in early diastole, during the so-called supernormal phase of excitation. This finding suggests either movement of the catheter tip with better electrode contact at the end of systole, or the development of 


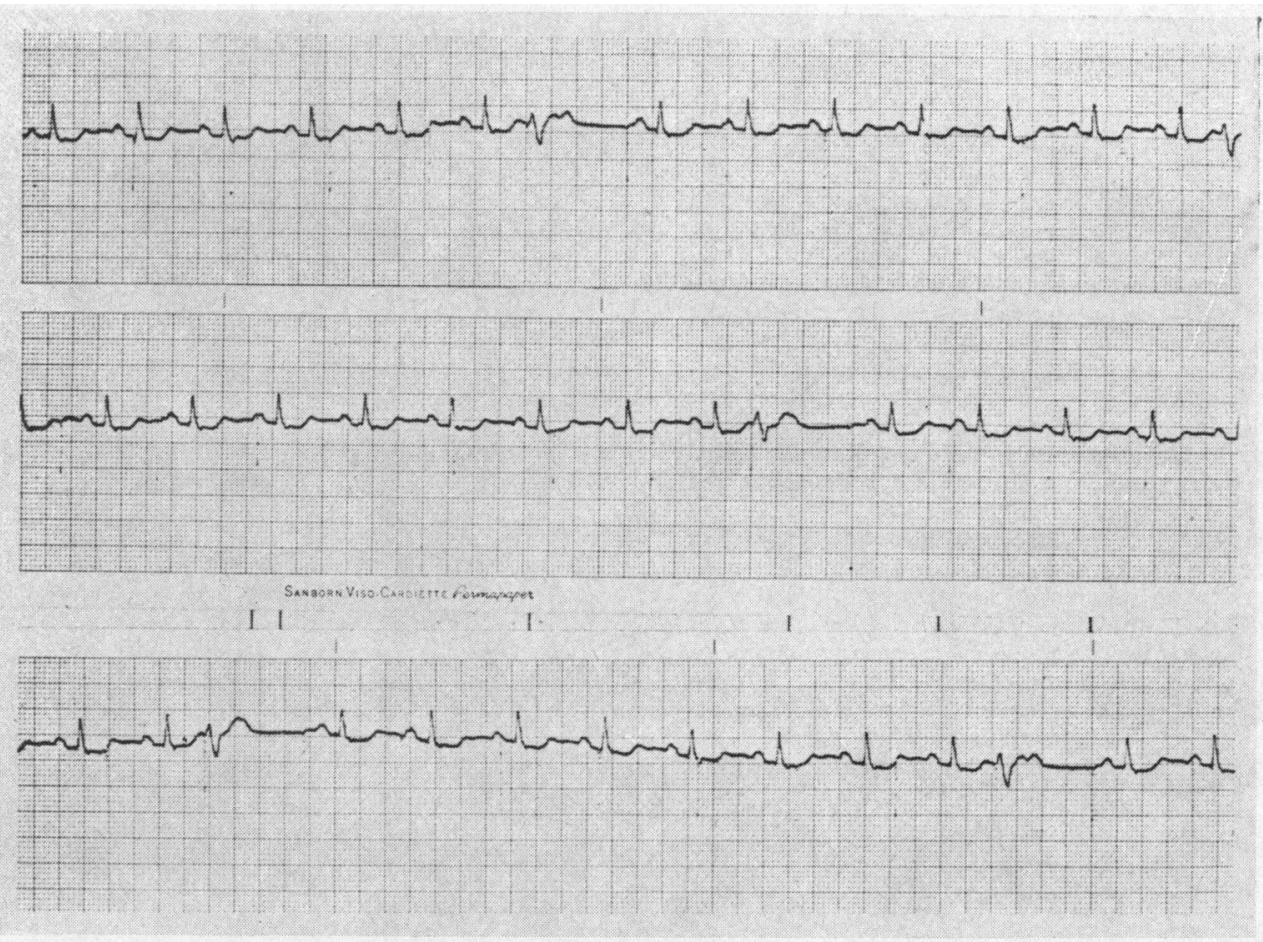

FIG. 4.-High-grade incomplete pacemaker capture in a patient with an implanted transvenous pacemaker and an abnormal increase in the excitation threshold. Sinus rhythm is present at 85 a minute and the transvenous pacemaker artefacts are evident at a rate of 75 a minute. Pacemaker captures occur only when the spike potentials fall 300 to 360 msec. after a preceding QRS depolarization (beats 7 and 15 top row, beat 10 middle row, and beats 3 and 12 bottom row), i.e. during the so-called supernormal phase of excitability of a preceding depolarization. (Lead II, see text for details.)

an increased stimulation threshold in association with fibrosis about chronically implanted endocardial electrodes (Lagergren et al., 1966). Either of these two explanations may account for pacemaker capture of the ventricle only on the $T$ wave, since the threshold for pacing is lowest during early repolarization (Hoffman and Cranefield, 1964). This phenomenon of supernormality for pacemaker stimulation was originally described by Linenthal and Zoll (1962) with implanted myocardial electrodes.

Fixed rate pacemaker generated rhythms represent an experimental model of naturally occurring parasystole. The concept of parasystole as described by Fleming (1912) depends on an isolated area of the myocardium which is not penetrated by, and automatically discharges without regard to, normally conducted impulses. The expression of these impulses, electrocardiographically, depends upon the ability of the ventricle to respond, i.e. the impulses must arrive when the ventricle is not refractory. The concept of parasystole was further advanced by the postulation of an exit block (Kauf- mann and Rothberger, 1919). This intermittent block prevents the emergence of impulses from the parasystolic centre to the surrounding myocardium. The basic rhythmicity of the parasystolic focus is, however, expressed at the same rate in subsequent beats. Occasionally, the manifest cycle of the parasystolic focus is irregular, and Pick (1953) postulated the existence of varying degrees of delayed conduction, or latency, of impulses from the ectopic centre to the remainder of the heart. The present observations, which document the existence of pacemaker latency and incomplete pacemaker capture during the administration of antiarrhythmic agents, appear to support Pick's postulation.

\section{SUMMARY}

Fixed rate pacemakers are a unique type of ventricular parasystole, and in the present study 4 varieties of incomplete pacemaker capture were observed in 5 of 129 patients with transvenous and transthoracic pacemakers. Examples of pacemaker 
latency, incomplete pacemaker capture with a Wenckebach type of myocardial conduction, isolated dropped beats, 2:1 capture, and high-grade incomplete capture are presented. All 5 patients had significant intrinsic myocardial disease. Three of the 5 patients were receiving antiarrhythmic agents which may have further altered the electrophysiological properties of the myocardium and contributed to the conduction disturbance. Two patients with implanted transvenous pacemakers had a late rise in the threshold for stimulation, suggesting either poor electrode contact or fibrosis about the catheter tip. These observations on pacemaker latency and incomplete pacemaker capture are discussed in terms of certain theories relating to ventricular parasystole.

\section{REFERENCES}

Chardack, W. M., Gage, A. A., and Greatbatch, W. (1960). A transistorized, self-contained, implantable pacemaker for the long-term correction of complete heart block. Surgery, 48, 643.
Fleming, G. B. (1912). Triple rhythm of the heart due to ventricular extra-systoles. Quart. F. Med., 5, 318.

Hoffman, B. F., and Cranefield, P. F. (1964). The physiological basis of cardiac arrhythmias. Amer. $\mathcal{F}$. Med., $37,670$.

Kaufmann, R., and Rothberger, C. J. (1919). Beiträge zur Entstehungsweise extrasystolischer, Allorhythmien (Zweite Mitteilung). Z. ges. exp. Med., 7, 199.

Lagergren, H., Johansson, L., Schüller, H., Kugelberg, J., Bojs, G., Alestig, K., Linder, E., Borst, H. G., Schaudig, A., Giebel, O., Harms, H., Rodewald, G., and Scheppokat, K. D. (1966). 305 cases of permanent intravenous pacemaker treatment for Adams-Stokes syndrome. Surgery, 59, 494.

Linenthal, A. J., and Zoll, P. M. (1962). Quantitative studies of ventricular refractory and supernormal periods in man. Trans. Ass. Amer. Phycns, 75, 285.

Medtronic, Inc. (1965). Implantable cardiac pacemaker instruction manual, Model 5870-c/5816. Minneapolis, Minnesota.

Mobitz, W. (1928). Über den partiellen Herzblock. Z. klin. Med., 107, 449.

Núñez-Dey, D., Zalter, R., and Eisenberg, H. (1962). Artificially induced parasystole in man due to surgically implanted myocardial pacemaker. Amer. F. Cardiol., 10, 535 .

Pick, A. (1953). Parasystole. Circulation, 8, 243. 